\title{
Efficacy of Local use of Metronidazole Solution among Acute Appendicitis Patients for the Prevention of Wound Infection after Appendectomy: A Randomized Control Trial
}

\author{
ABM Mir Mubinul Islam¹, Nasima Akhter², Md. Zafar Imam³, Mafiur Rahman \\ Jalal Uddin Mohammad Rumi ${ }^{5}$

\begin{abstract}
${ }^{1}$ Senior Consultant (Surgery), District Hospital, Joypurhat, Bangladesh; ${ }^{2}$ Consultant (Gynaecology \& Obstetrics), District Hospital, Joypurhat, Bangladesh; ${ }^{3}$ Consultant (Orthopaedics), District Hospital, Joypurhat, Bangladesh; ${ }^{4}$ Senior Consultant (Surgery), District Hospital, Joypurhat, Bangladesh; ${ }^{5}$ Assistant Professor, Department of Neurosurgery, National Institute of Neurosciences \& Hospital, Bangladesh
\end{abstract}

[Received: 12 August 2019; Accepted: 20 October 2019; Published: 1 January 2020]

\begin{abstract}
Background: Prevention of wound infection after appendectomy is very important for the post-operative management of the acute appendicitis patients. Objective: The purpose of the present study was to validate the efficacy of local application of metronidazole solution in the wound after closing of peritoneum in the prevention of appendectomy wound infection. Methodology: This randomized control trial was conducted in the Department of Surgery at Rajshahi Medical College Hospital, Rajshahi, Bangladesh from July 2009 to June 2011 for a period of two years. Patients admitted with features of uncomplicated and complicated acute appendicitis (Gangrenous or perforated) underwent emergency appendectomy by grid iron incision in all the units of surgery were selected as study population. The study population were divided into two groups into group A and group B. In group A (treatment group) after closure of peritoneum the exposed tissue was washed and was flooded with $100 \mathrm{ml}$ of metronidazole solution with $5 \mathrm{gm} / \mathrm{ml}$ concentration and was waited for one minute and in group B (control group) with the same amount of sterile saline solution. All patients were evaluated daily for evidence of wound infection such as fever, unusual wound pain with recording of pulse, temperature. Suspected wounds were checked on 3rd, 5th and 7th POD routinely after removal of dressing. Result: A total number of 200 patients were recruited for this study and was divided into two groups in equal number. Therefore, 100 cases were in group A and the rest of 100 cases were enrolled in group B. The mean age with SD of group A and B were 25.06 \pm 9.14 years and $23.82 \pm 7.51$ years respectively. Uncomplicated appendicitis was found in 66 cases and 70 cases in group A and B respectively ( $>0.05)$. In metronidazole wound wash group only 5 patients developed wound infection, however, in saline wash group 22 patients developed infection. Among the positive cultures Staphylococcus aureus was 3 cases and Escherichia coli was in 7 cases. Conclusion: In conclusion rate of wound infection is less in the group treated with the local application of metronidazole solution after appendectomy. [Journal of National Institute of Neurosciences Bangladesh, 2019;6(1):54-58]

Keywords: Post-operative; local use; metronidazole solution; wound infection; appendectomy patients

Correspondence: Dr. ABM Mir Mubinul Islam, Senior Consultant (Surgery), District Hospital, Joypurhat, Bangladesh; Email: abmmubinul@gmail.com; Cell no.: +8801716696444

Conflict of interest: There is no financial conflict of interest relevant to this paper to disclose.

Funding agency: This research project was not funded by any group or any institution.

Contribution to authors: Islam ABMMM, Akhter N, Imam MZ involved in protocol preparation, data collection and literature search up to manuscript writing. Rahman M, Rumi JUM involved in preparation and revision of this manuscript.

How to cite this article: Islam ABMMM, Akhter N, Imam MZ, Rahman M, Rumi JUM. Efficacy of Local use of Metronidazole Solution among Acute Appendicitis Patients for the Prevention of Wound Infection after Appendectomy: A Randomized Control Trial. J Natl Inst Neurosci Bangladesh, 2020;6(1):54-58

Copyright: (C2020. Islam et al. Published by Journal of National Institute of Neurosciences Bangladesh. This article is published under the Creative Commons CC BY-NC License (https://creativecommons.org/licenses/by-nc/4.0/). This license permits use, distribution and reproduction in any medium, provided the original work is properly cited, and is not used for commercial purposes.
\end{abstract}

\section{Introduction}

Appendectomy remains one of the most common abdominal operations ${ }^{1}$. However, relative to other frequent operations, such as, cholecystectomy and hysterectomy, it has the highest rate of post-operative infectious complications ${ }^{2}$. Therefore it is an excellent model to investigate surgical site infections (SS1S) in abdominal operations ${ }^{3}$.

Infection rate has been reported varying from $10 \%$ to $30 \%$ cases $^{4}$. The presence of bacteria in the incision at the time of wound closure is the most important factor in wound sepsis. The predominant microbial 
flora associated with acute appendicitis are Escherichia coli, Klebsiella species, Proteus species and Bacteriodes fragilis 5 . Bacteriodes fragilis to be the commonest organism in infected appendectomy wounds, and a survey has showed anaerobic organisms more often than aerobes ${ }^{6}$. However, anaerobic sepsis has been abolished by metronidazole. These organisms may cause post-operative wound infection depending upon the degree of inflammation of appendix, the technique of surgery, the duration of operation, and which are the important factors for the risk of developing wound infection ${ }^{7}$. Among the antimicrobial agents available for the treatment of and as well as prophylaxis against anaerobic infections, the nitro imidazoles like metronidazole, ornidazole and tinidazole have gained considerable application as potent, nontoxic substances with a narrow spectrum ${ }^{8}$.

For wound infections caused by aerobic organism like Escherichia coli, Klebsiella species ampicillin cephalosporins, quinolones and aminoglycosides are available for both the treatment and prophylaxis ${ }^{8}$. These antibiotics in combination covering both aerobes and anaerobes have been being used enterally or parenteral as the treatment and prophylaxis of wound infection after appendectomy. Systemic usages of antibiotics have reduced the rate of infection but they are far from satisfaction. Alternative methods of antibiotic delivery have been studied ${ }^{9}$.

In vitro studies demonstrated $100 \%$ kill rates with clinically usable concentrations of antimicrobials in irrigating solutions after only 60 -seconds exposure of the organisms. Thus, metronidazole administrated rectally or intravenously or locally has been reported to be of value in the prevention and treatment of infection after appendectomy ${ }^{10}$. Therefore, this present study was undertaken to validate the efficacy of local application of metronidazole solution in the wound after closing of peritoneum in the prevention of appendectomy wound infection.

\section{Methodology}

Study Population and Settings: This single centre, parallel, randomized control trial was conducted in the Department of Surgery, Rajshahi Medical College Hospital, Rajshahi, Bangladesh from July 2009 to June 2011 for a period of two years. Patients admitted with features of uncomplicated and complicated acute appendicitis (Gangrenous or perforated) underwent emergency appendectomy by grid iron incision in all the units of surgery were selected as study population. Study population was first screened by Modified Alvarado score. Patients with appendectomies which was not done by grid iron incision, patients underwent interval or incidental appendectomy, histologically negative ones and patients below the age of 13 years or patients who had receiving steroid, cytotoxic or any immunosuppressive drugs were excluded from this study. All patients underwent appendectomy by Grid Iron incision were included. Approval from the Institutional Review Board (IRB) of Rajshahi Medical College was taken prior to the study. The purpose of study was explained to the patients and informed consent was taken. All clinical information including history, physical findings and investigation reports were collected and recorded in a pre-designed data collection sheet.

Randomization \& Blinding: The study population were divided into two groups based on computer generated simple random sampling technique designated as group A and Group B. Blinding was not done.

Allocation: In group A (treatment group) after closure of peritoneum the exposed tissue was washed and was flooded with $100 \mathrm{ml}$ of metronidazole solution with $5 \mathrm{gm} / \mathrm{ml}$ and was waited for one minute (Scherr DD, Dodd TA) and in group B (control group) with the same amount of sterile saline solution. Simply inflamed or doubtfully inflamed appendix were sent for histopathological examination and the negative cases were excluded from the study. Pus or swabs from the infected wounds were sent for culture and sensitivity. All patients were given preoperative intravenous ciprofloxacin and metronidazole in adult doses. When the patients started oral feeding the antibiotics were switched over to oral form. It was continued for 7 days post operatively. Per operatively aseptic and antiseptic measures, operative techniques were followed according to the standard. Pre-operatively informed consent after explanation of purpose of the study was taken from all the patients under investigation.

Follow up and Outcome measures: All patients were evaluated daily for evidence of wound infection such as fever, unusual wound pain with recording of pulse, temperature. Suspected wounds were checked on $3^{\text {rd }}, 5^{\text {th }}$ and $7^{\text {th }}$ POD routinely after removal of dressing. Otherwise wound was left undisturbed. Postoperatively all of the patients were followed up in in-patient and out-patient department of surgery in 
$15^{\text {th }}$ and $30^{\text {th }}$ day after operation for identifying any evidence of wound infection.

Statistical Analysis: Collected data were analyzed by appropriate statistical test and it was processed by SPSS version 22.0 (Statistical Package for Social Science) software. Qualitative data were expressed as frequency and percent. Quantitative data were expressed as mean with standard deviation. The comparison of qualitative data was performed by Chi square test and the quantitative data were by Student $t$ test. $\mathrm{P}$ value less than 0.05 was taken as statistically significant.

\section{Results}

A total number of 200 patients were recruited for this study and was divided into two groups in equal number. Therefore, 100 cases were in group A and the rest of 100 cases were enrolled in group B. Wounds were closed in layers without drainage and same type of suture materials were used. Both uncomplicated and complicated appendicitis (gangrenous/perforated) were included. In 13 to 20 years of age group $44(44.0 \%)$ cases and $40(40 \%)$ cases were present in group A and B respectively. However, $30(30.0 \%)$ cases and $46(46.0 \%)$ cases were present in 21 to 30 years of age group. The mean age with SD of group A and B were $25.06 \pm 9.14$ years and $23.82 \pm 7.51$ years respectively (Table 1).

Table 1: Age Distribution in Group A and Group B

\begin{tabular}{lccc}
\hline Age Group & Group A & Group B & P value \\
\hline 13 to 20 Years & $44(44.0 \%)$ & $40(40 \%)$ & \\
21 to 30 Years & $30(30.0 \%)$ & $46(46.0 \%)$ & $0.067^{*}$ \\
31 to 40 Years & $22(22.0 \%)$ & $12(12.0 \%)$ & \\
More than 40 Years & $4(4.0 \%)$ & $2(2.0 \%)$ & \\
Total & $\mathbf{1 0 0 ( 1 0 0 . 0 \% )}$ & $\mathbf{1 0 0}(\mathbf{1 0 0 . 0 \% )}$ & \\
Mean age & $25.06( \pm 9.14)$ & $23.82( \pm 7.51)$ & $0.296^{* *}$ \\
\hline
\end{tabular}

*Chi square test was performed to see the level of significance;

**Student $t$ test was performed to see the level of significance

Uncomplicated appendicitis was found in 66 cases and 70 cases in group A and B respectively. However, complicated appendicitis was found in 34 cases and 30 cases in group A and B respectively (Table 2).

Table 2: Status of Acute Appendicitis in Group A and Group B

\begin{tabular}{lccc}
\hline Status of Appendicitis & Group A & Group B & P value \\
\hline Uncomplicated & $66(66.0 \%)$ & $70(70.0 \%)$ & \\
Complicated & $34(34.0 \%)$ & $30(30.0 \%$ & 0.325 \\
Total & $\mathbf{1 0 0 ( 1 0 0 . 0 \% )}$ & $\mathbf{1 0 0 ( 1 0 0 . 0 \% )}$ & \\
\hline
\end{tabular}

*Chi square test was performed to see the level of significance
In metronidazole wound wash group only 5 patients developed wound infection, however, in saline wash group 22 patients developed infection. Again, wound infection was absent in 95 cases and 78 cases in group A and group B respectively (Table 3).

Table 3: Distribution of Wound Infection among Study Population

\begin{tabular}{lccc}
\hline Wound Infection & Group A & Group B & P value \\
\hline Present & $5(5.0 \%)$ & $22(22.0 \%)$ & \\
Absent & $95(95.0 \%)$ & $78(78.0 \%)$ & 0.001 \\
Total & $\mathbf{1 0 0 ( 1 0 0 . 0 \% )}$ & $\mathbf{1 0 0}(\mathbf{1 0 0 . 0} \%)$ & \\
\hline
\end{tabular}

*Chi square test was performed to see the level of significance

No growth was found in 17 cultures out of 27 cultures. Among the positive cultures Staphylococcus aureus was 3 cases and Escherichia coli was in 7 cases (Table 4).

Table 4: Results of Bacterial Culture in Group A and Group B

\begin{tabular}{lccc}
\hline Bacterial Culture & Group A & Group B & Total \\
\hline Staph. aureus & 1 & 2 & 3 \\
E. coli & 3 & 4 & 7 \\
No growth & 1 & 16 & 17 \\
Total & $\mathbf{5}$ & $\mathbf{2 2}$ & $\mathbf{2 7}$ \\
\hline
\end{tabular}

\section{Discussion}

There are many studies on the topical use of antiseptics, antibiotics either narrow or broad spectrum, to prevent post-operative wound infection, and which have been shown to be highly effective ${ }^{8-10}$. Local use of antibiotics has immediate effect and provides higher concentration of antibiotic than that could be achieved by systemic ones. Exposure of organisms to this higher concentration only for one minute (60 seconds) can kill them instantly ${ }^{11}$. In gut surgeries where anaerobic pathogens are the main organisms to cause wound infection. Use of metronidazole solution locally in adjunct to systemic use to combat anaerobic infection has been tried ${ }^{12}$.

Acute appendicitis was diagnosed by Modified Alvarado Score. All patients of uncomplicated and complicated appendicitis (Gangrenous/perforated) underwent appendectomy by Grid Iron incision were included. Simply inflamed or doubtfully inflamed appendices were sent for histopathological examination and the negative cases were excluded from the study. Pus/swabs from the infected wounds were sent for culture and sensitivity. All patients were given preoperative intravenous ciprofloxacin and metronidazole in adult doses. When the patients took 
oral feeding the antibiotics were switched over to oral form. It was continued for 7 days post operatively.

The operations were performed by different mid-level trainee surgeons with varying degrees of experience and skill. Most of the operations done in emergency OT in the evening and at night. In this series 136(68\%) patient were suffering uncomplicated appendicitis and $64(32 \%)$ patents were complicated. In locally metronidazole treated group complicated appendicitis $34(34 \%)$ and in the control group complicated appendicitis $30(30 \%)$.

In the interventional (metronidazole wound wash given) group only 5(5\%) patients developed wound infection where as in the control (metronidazole not given locally) group 22(22\%) patients developed wound infection. Here p value is 0.001 which is highly statistically significant. Culture of pus or swabs from the infected wounds have showed no growth in 17 cultures out of 27 cultures. Among the positive cultures Staphylococcus aureus is found in 3 cases and Escherichia coli is in 7 in number. Most of the cases Escherichia coli is found to be resistant to ciprofloxacin and ceftriaxone ${ }^{13}$. They were sensitive to amoxicillin, gentamycin and imepenem.

These findings can be compared with the study of El-Sefi et $\mathrm{al}^{14}$ showed that appendectomy was performed on 100 patients with complicated appendicitis through a grid-iron incision. All patients received systemic metronidazole and cephazolin sodium which started preoperatively and continued postoperatively for 5 days. At operation, patients were allocated randomly to receive either local instillation of metronidazole and cephazolin intraperitoneally and interparietally (group A) or no local antibiotic therapy (group B). The conclusion of this study is that a single peroperative instillation of metronidazole and cephazolin into the peritoneum and wound layers is a safe and valuable adjunct to the perioperative systemic administration of these drugs in significantly reducing postoperative sepsis and duration of hospital stay in complicated appendicitis.

Parcells et al ${ }^{15}$ performed a retrospective study of adult appendectomies at a single institution They sought to determine whether there was a difference in postoperative surgical site infections (SSIs) comparing normal saline, antiseptic solution and an antibiotic solution (imipenem $1 \mathrm{mg} / \mathrm{mL}$ ). Saline has an SSI rate of $9.8 \%$, a wound infection rate of $7.3 \%$, and an abdominal abscess rate of $4.2 \%$. Imipenem irrigation has an SSI rate of $0.5 \%$, a wound infection rate of $0.5 \%$ and an abdominal abscess rate of $0.5 \%$. These results suggest that abdominal irrigation with an antibiotic solution is superior to both normal saline. This study supports the local use of metronidazole solution.

Shubing and Litian ${ }^{16}$ have demonstrated that precisely $0.915 \mathrm{~g}$ metronidazole disodium phosphate injection or $25 \%$ metronidazole glucose solution has been added to $100 \mathrm{~mL} 0.9 \%$ normal saline. After anesthetizing the patients 60 to $80 \mathrm{~mL}$ of the solution has been injected into the subcutaneous tissue and muscle. The control group has been given intravenous injection of metronidazole disodium phosphate and cephazolin postoperatively. In the treated group the rate of incision infection is $0.8 \%$. By contrast, the rate of infection among control group is $11.6 \%$ cases. The statistical analysis is significant $(\mathrm{p}=0.001)$ showing that preincisional or intraincisional infiltration with metronidazole is effective.

In another study ${ }^{17}$ it has been reported that after suture of the peritoneum, the exposed tissue washed with tinidazole solution significantly has reduced post-operative wound infection in cases of gangrenous appendicitis and it was highly cost effective. The study is prospective, controlled, and double-blind. The total number of postoperative wound infections is 3.5\% cases. Among 116 patients with gangrenous appendicitis, a significant reduction of postoperative wound infections is observed in the group given tinidazole prophylaxis $(\mathrm{p}=0.02)$. The infection rates observed is $2.0 \%$ in the treatment group and $13.8 \%$ in the control group. Local application of tinidazole in cases of gangrenous appendicitis has thus proved to be highly effective. They have suggested local application of tinidazole to reduce appendectomy wound infection. With the advent of new generation parenteral antibiotics, the modern trend in general surgery is to use prophylactic antibiotic parenteral ${ }^{7}$. The emergence of antibiotic resistance has become a great concern to all and alternative and short term use of antibiotic is being studied. Most of the studies concluded that therapeutic effect of metronidazole solution either by infiltration or instillation in prevention of appendectomy wound infection was reliable and no side effect was found ${ }^{11}$.

However, there are some limitations of this present study. Only routine culture of infected wounds has been done. Culture for anaerobic organisms could not be done due to lack of facilities. Histopathology of all the resected appendix could not be done. Appendectomies are done by different trainee surgeons with varying degrees of experience and skill. With the above limitations it can be suggested that local use of 
metronidazole solution either by infiltrating the wound area or by simple wash of the exposed wound tissue by the solution has significant role in reducing appendectomy wound infection.

\section{Conclusion}

Role of prophylactic antibiotics in surgical practice, especially in cases where contamination is inevitable, is well documented. A prophylactic antibiotic is not an alternative to good surgical practice which includes antisepsis, disinfection, gentle tissue handling, avoidance of dead space, tensionless suturing, sterilization techniques, etc.

From this small study it is anticipated that washing the wound with metronidazole solution is better than washing it with normal saline. Culture of pus from majority of the infections in the control group showed no growth. In the Case group, therefore, metronidazole wound wash has reduced the rate of wound infection. Wound infection would be reduced further if the wounds could be washed with an antibiotic covering both aerobes and anaerobes.

\section{References}

1. Eklund AE, Tunevall TG. Prevention of postoperative wound infection after appendectomy by local application of tinidazole: a double-blind study. World journal of surgery. 1987 Apr 1;11(2):263-6.

2. Ravari H, Jangjoo A, Motamedifar J, Moazzami K. Oral metronidazole as antibiotic prophylaxis for patients with nonperforated appendicitis. Clinical and experimental gastroenterology. 2011:4:273.

3. Krukowski ZH, Irwin ST, Denholm S, Matheson NA. Preventing wound infection after appendicectomy: a review. British journal of surgery. 1988 Oct;75(10):1023-33.

4. Ravari H, Jangjoo A, Motamedifar J, Moazzami K. Oral metronidazole as antibiotic prophylaxis for patients with nonperforated appendicitis. Clinical and experimental gastroenterology. 2011;4:273.

5. Ein SH, Sandler A. Wound infection prophylaxis in pediatric acute appendicitis: a 26-year prospective study. Journal of pediatric surgery. 2006 Mar 1;41(3):538-41.
6. McGreal GT, Joy A, Manning B, Kelly JL, O'Donnell JA, William W, Kirwan O, Redmond HP. Antiseptic wick: does it reduce the incidence of wound infection following appendectomy?. World journal of surgery. 2002 May 1;26(5):631-4.

7. Fitzmaurice GJ, McWilliams B, Hurreiz H, Epanomeritakis E. Antibiotics versus appendectomy in the management of acute appendicitis: a review of the current evidence. Canadian journal of Surgery. 2011 Oct;54(5):307.

8. Charalambous C, Tryfonidis M, Swindell R, Lipsett AP. When should old therapies be abandoned? A modern look at old studies on topical ampicillin. Journal of Infection. 2003 Oct 1;47(3):203-9.

9. Hesami MA, Alipour H, Daylami HN, Alipour B, Bazargan-Hejazi S, Ahmadi A. Irrigation of abdomen with imipenem solution decreases surgical site infections in patients with perforated appendicitis: a randomized clinical trial. Iranian Red Crescent Medical Journal. 2014 Apr;16(4).

10. Tijerina J, Velasco-Rodríguez R, Vasquez C, Melnikov V, Rodriguez S. Effectiveness of a systemic antibiotic followed by topical ionized solution as surgical site infection prophylaxis. Journal of International Medical Research. 2010 Aug;38(4):1287-93.

11. Bhargava P, Mehrotra N, Kumar A. Wound infection after metronidazole infiltration. Tropical doctor. 2006 Jan 1;36(1):37-8. 12. Cervantes-Sánchez CR, Gutiérrez-Vega R, Vázquez-Carpizo JA, Athié-Gutiérrez C. Syringe pressure irrigation of subdermic tissue after appendectomy to decrease the incidence of postoperative wound infection. World journal of surgery. $2000 \mathrm{Jan}$ $1 ; 24(1): 38-42$

13. El-Sefi TA, el-Awady HM, Shehata MI, al-Hindi MA. Systemic plus local metronidazole and cephazolin in complicated appendicitis: a prospective controlled trial. J R Coll Surg Edinb. $1989 \mathrm{Feb} ; 34(1): 13-6$.

14. Jangjou A, Amouzeshi A, Kavianifar K, Mehrabi BM. Wound infection incidence in patients with simple and gangrenous or perforated appendicitis.

15. Parcells JP, Mileski JP, Gnagy FT, Haragan AF, Mileski WJ. Using antimicrobial solution for irrigation in appendicitis to lower surgical site infection rates. The American journal of surgery. 2009 Dec 1;198(6):875-80

16. Shubing W, Litian Z. Preventing infection of the incision after appendectomy by using metronidazole preoperatively to infiltrate tissue at the incision. Am J Sur, 1997;174 (4), pp. 422-24

17. Lin KB, Lai KR, Yang NP, Chan CL, Liu YH, Pan RH, Huang $\mathrm{CH}$. Epidemiology and socioeconomic features of appendicitis in Taiwan: a 12-year population-based study. World Journal of Emergency Surgery. 2015 Dec;10(1):42 